\title{
Laboratory Simulation of Chemical Reactions in Interstellar Ices
}

\author{
K. Hiraoka, T. Sato, and T. Takayama \\ Faculty of Engineering, Yamanashi Univ., Takeda-4, Kofu 400, Japan
}

\begin{abstract}
The reactions of $\mathrm{H}$ atoms with solid thin films at $10 \mathrm{~K}$ were studied by using thermal desorption mass spectrometry and FT-IR spectroscopy. The $\mathrm{N}, \mathrm{C}$, and $\mathrm{O}$ atoms trapped in solid matrices were converted efficiently to fully hydrogenated compounds. In the reaction of $\mathrm{H}$ atoms with a solid CO film, the formation of formaldehyde and methanol were confirmed. The relatively low yield of the reaction products suggests either the smaller rate constants of the $\mathrm{H}$ atom addition reactions to $\mathrm{CO}$ and/or the occurrence of the hydrogen abstraction reaction $\mathrm{H}+\mathrm{HCO}$ $\rightarrow \mathrm{H}_{2}+\mathrm{CO}$. The reactions of $\mathrm{H}$ atoms with thin films of acetone and 2-propanol were also studied. The major products from acetone were found to be methane and alcohols but 2-propanol was not detected as a reaction product. The reaction of $\mathrm{H}$ with 2-propanol led to the formation of methane, alcohols, and acetone as major products.

In the reaction of $\mathrm{H}$ with $\mathrm{C}_{2} \mathrm{H}_{2}, \mathrm{C}_{2} \mathrm{H}_{6}$ was found to be the major product but $\mathrm{C}_{2} \mathrm{H}_{4}$ could not be detected. This is due to the fact that the first-step addition reaction $\mathrm{H}+\mathrm{C}_{2} \mathrm{H}_{2} \rightarrow \mathrm{C}_{2} \mathrm{H}_{3}$ is the rate-controlling process and the following reactions to form the final product $\mathrm{C}_{2} \mathrm{H}_{6}$ proceed much faster than the initial one. This finding is in accord with the observation of comets Hyakutake and Hale-Bopp, i.e., $\mathrm{C}_{2} \mathrm{H}_{2}$ and $\mathrm{C}_{2} \mathrm{H}_{6}$ but not $\mathrm{C}_{2} \mathrm{H}_{4}$ were detected in the coma of these comets. In the reactions of $\mathrm{H}$ with $\mathrm{C}_{2} \mathrm{H}_{2}$ and $\mathrm{C}_{2} \mathrm{H}_{4}$, the $\mathrm{C}_{2} \mathrm{H}_{6}$ product yields increased drastically with decrease of temperature from 50 to $10 \mathrm{~K}$. This is most likely due to the increase of the sticking probability of $\mathrm{H}$ atoms on the solid films at lower temperature. These findings led us to conclude that the chemical evolution taking place on the dust grains via $\mathrm{H}$-atom tunneling reactions becomes efficient only at cryogenic temperatures, i.e., $\sim 10 \mathrm{~K}$.
\end{abstract}

\section{Introduction}

The evidence that grain chemistry occurs in the interstellar medium (Williams \& Millar 1993) has been accumulated and models which examined the interaction between grain surface processes with a gas-phase chemistry have been proposed (e.g. Tielens \& Hagen 1982; d'Hendecourt, Allamandola, \& Greenberg 1985; Hasegawa, Herbst, \& Leung 1992). Despite the wealth of data on gas-phase ionmolecule reactions (Ikezoe et al. 1987), investigations of the solid phase reactions are rather scarce (Greenberg \& Pirronello 1989). The Leiden group has made laboratory simulations of the effects of UV irradiation on the icy grains (Hong \& 
Greenberg 1980; Greenberg \& Pirronello 1989; Greenberg \& Li 1996; Gerakines, Schutte, \& Ehrenfreund 1996). Pirronello et al. performed laboratory synthesis of molecular hydrogen on surfaces of astrophysical interest (Pirronello et al. 1997a,b, 1999). Roessler and co-workers made theoretical and laboratory studies of the interaction of cosmic-ray particles with interstellar ices (Kaiser \& Roessler 1997, 1998; Kaiser et al. 1997, 1999).

Our laboratory has made continuing efforts to elucidate the chemical evolution taking place on dust grains in dark clouds at 10-20 K. Our main research objective is to clarify the tunneling reaction mechanisms of $\mathrm{H}$ atoms with major interstellar molecules and to evaluate the roles of tunneling reactions in the chemical evolution on the dust grains in dark clouds.

\section{Experimental}

The experimental procedures are similar to those described in our previous papers (Hiraoka et al. 1994, 1995, 1998a,b, 1999). The conceptual idea for the apparatus is shown in Figure 1. The cryocooler (Iwatani Plantech, type D310) and a quadrupole mass spectrometer (Leda Mass, Microvision 300D) were housed in a vacuum manifold. The base pressure of the vacuum system under the current experimental conditions was $\sim 5 \times 10^{-10}$ Torr after baking the vacuum system.

The sample gases were deposited on the silicon substrate ((100) surface with a size of $30 \times 50 \times 0.5 \mathrm{~mm}$ ) which was pressed firmly to the cold head of the cryocooler using indium foil between the mating surfaces. After the deposition of the sample, the sample film was sprayed by $\mathrm{H}$ atoms $\left(\sim 27 \mathrm{~K}\right.$, flux of $10^{13}$ $10^{14} \mathrm{~cm}^{-2} \mathrm{~s}^{-1}$ ) produced by the dc discharge plasma of $\mathrm{H}_{2}$ gas in the bottle-neck discharge tube. The film was completely prevented from being bombarded by the charged particles and UV photons produced by the plasma (see Hiraoka et al. 1998b). It was confirmed that the local heating and resultant desorption of molecules from the surface of the sample was negligible when the $\mathrm{H}$ atomcontaining $\mathrm{H}_{2}$ gas was sprayed over the deposited sample (Hiraoka et al. 1998b).

Infrared spectra of products formed from the reaction of $\mathrm{H}$ atoms with deposited samples were measured using a FT-IR spectrometer (Nicolet, MagnaIR 760) with a resolution of $4 \mathrm{~cm}^{-1}$ in combination with a $\mathrm{KBr}$ beam splitter and a liquid $\mathrm{N}_{2}$ cooled $\mathrm{MCT}(\mathrm{HgCdTe})$ detector.

\section{Results and Discussion}

In diffuse interstellar clouds, ion-molecule reactions are the dominant processes for the molecular formation. Because the temperature of the dark cloud is $\sim 10$ $\mathrm{K}$, all gases except for $\mathrm{He}, \mathrm{Ne}$, and $\mathrm{H}_{2}$ may be condensable on the surfaces of dust grains and form mantles on the dust. In order to elucidate the chemical evolution of dark clouds, it is important to investigate the chemical processes taking place on the surfaces of dust grains and in their mantles. Figure 2 summarizes the possible processes for the formation of $\mathrm{NH}_{3}, \mathrm{CH}_{4}, \mathrm{H}_{2} \mathrm{O}, \mathrm{H}_{2} \mathrm{CO}$ and $\mathrm{CH}_{3} \mathrm{OH}$, i.e., $\mathrm{N}, \mathrm{C}, \mathrm{O}$ atoms and $\mathrm{CO}$ molecules adsorbed on the dust grains are hydrogenated to fully hydrogenated compounds on the dust grains by the $\mathrm{H}$ atoms impinging on the dust grains. 


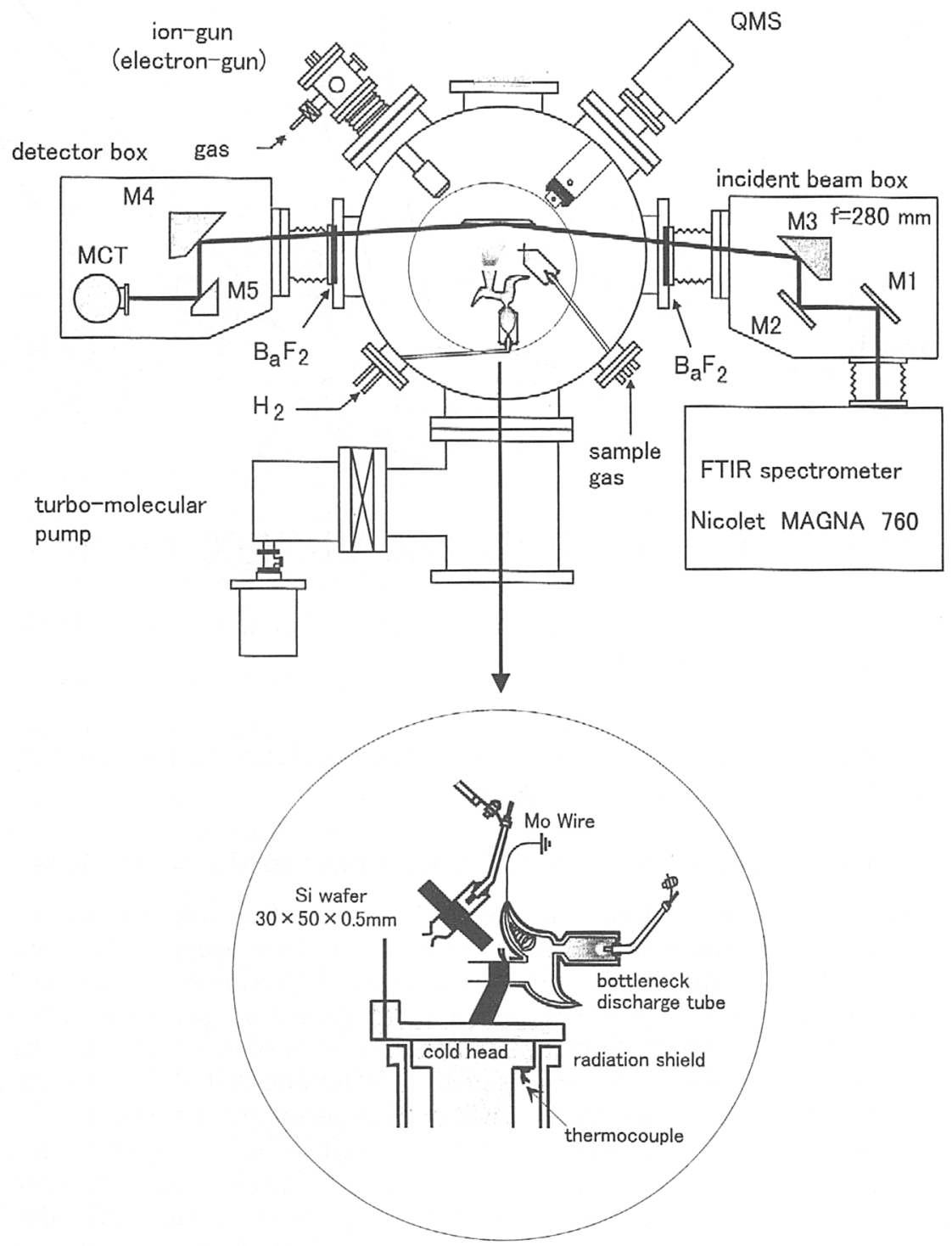

Figure 1. Schematic diagram of the experimental apparatus. 


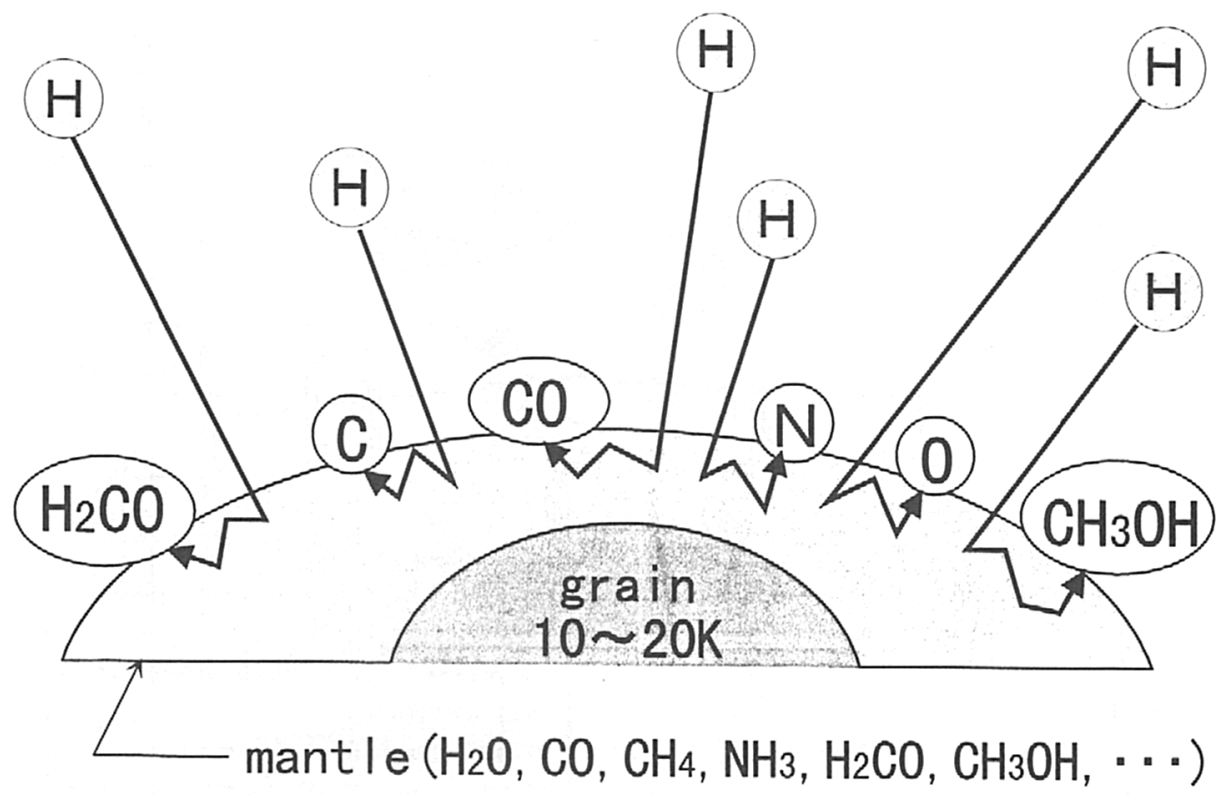

Figure 2. The conceptual idea for the formation of $\mathrm{NH}_{3}, \mathrm{CH}_{4}, \mathrm{H}_{2} \mathrm{O}$, $\mathrm{H}_{2} \mathrm{CO}$ and $\mathrm{CH}_{3} \mathrm{OH}$ on the cold dust grains.

In the following sections, the experimental evidence will be given which verifies the reactions proposed in Figure 2.

\subsection{Reactions of $\mathbf{H}$ atoms with $\mathrm{N}, \mathrm{C}$, and $\mathrm{O}$ in the solid phase}

If atoms other than $\mathrm{H}$ atoms hit the cold grain surface and are trapped in the grain mantles, the recombination reactions of these atoms with mobile $\mathrm{H}$ atoms may lead to the formation of hydrogenated products. In hot molecular cores the high $\mathrm{NH}_{3}$ abundance cannot be explained by gas-phase chemistry (Brown \& Charnley 1990). Furthermore, the presence of enhanced abundances of deuterated molecules in hot cores, such as $\mathrm{HDO}$ and $\mathrm{NH}_{2} \mathrm{D}$, is indicative of the importance of deuterium fractionation reactions on grain surfaces.

To begin with, the reactions of $\mathrm{H}$ atoms with $\mathrm{N}$ atoms trapped in a $\mathrm{N}_{2}$ matrix in the temperature range of $10-30 \mathrm{~K}$ have been studied in order to obtain information on the possibility of $\mathrm{NH}_{3}$ synthesis in the grain mantles (Hiraoka et al. 1995). It was found that the $\mathrm{H}$ atoms diffuse in the $\mathrm{N}_{2}$ matrix and hydrogenate $\mathrm{N}$ atoms trapped in the $\mathrm{N}_{2}$ matrix to $\mathrm{NH}_{3}$. By an electron spin resonance (ESR) experiment, $\mathrm{H}$ atoms were found to diffuse in $\mathrm{N}_{2}$ and $\mathrm{H}_{2} \mathrm{O}$ matrices at $\sim 10 \mathrm{~K}$. These experimental results suggest that the hydrogenation reactions on the dust grains play important roles in the chemical evolution in the interstellar medium.

It is known that formation of saturated hydrocarbons in the interstellar medium is difficult to explain by gas-phase ion-molecule reactions because the carbonium ions cease to react with $\mathrm{H}_{2}$ at the unsaturated forms. Consequently, 
observations of a high abundance of $\mathrm{CH}_{4}$, either frozen on grains or as a gas, would provide strong evidence that reactions on dust grains play an important role in interstellar chemistry. In order to verify the importance of grain chemistry for the formation of $\mathrm{CH}_{4}$, we investigated the reaction of $\mathrm{H}$ atoms with $\mathrm{C}$ atoms embedded in a solid $\mathrm{CO}$ matrix. It was found that $\mathrm{H}$ atoms migrate in the $\mathrm{CO}$ matrix and react with trapped $\mathrm{C}$ atoms selectively to form $\mathrm{CH}_{4}$. This indicates that $\mathrm{H}$ atoms are far less reactive with $\mathrm{CO}$ molecules than with $\mathrm{C}$ atoms and they can manage to migrate and encounter the $\mathrm{C}$ atoms to form $\mathrm{CH}_{4}$ in the $\mathrm{CO}$ matrix. It was also found that $\mathrm{D}_{2} \mathrm{O}$ was efficiently synthesized in the reaction of $\mathrm{D}$ atoms with $\mathrm{O}$ atoms embedded in the $\mathrm{N}_{2} \mathrm{O}$ matrix. In summary, the consecutive hydrogenation reactions of $\mathrm{N}, \mathrm{C}$, and $\mathrm{O}$ atoms trapped in the solid phase were found to proceed efficiently to fully hydrogenated compounds. These results may seem to be reasonable because the radical-radical recombination reactions are generally believed to have negligibly small energy barriers. However, it should be noted that in some cases the radical-radical recombination reactions could have small but non-negligible energy barriers even in the gas phase $(\sim 100 \mathrm{~K}$ or so). The energy barrier will increase in the solid phase reactions due to the rigid matrix effect. The observed efficient synthesis of the fully hydrogenated compounds $\mathrm{NH}_{3}, \mathrm{CH}_{4}$, and $\mathrm{H}_{2} \mathrm{O}$ at $\sim 10 \mathrm{~K}$ may be explained by the fact that the radical-radical recombination reactions have an early barrier in their reaction coordinates which are favored for the occurrence of exothermic reactions.

\subsection{Reaction of $\mathbf{H}$ atoms with solid $\mathbf{C O}$}

The significant abundance of formaldehyde in the diffuse envelopes of dark clouds cannot be explained by purely gas phase processes. After $\mathrm{H}_{2}, \mathrm{CO}$ is the most abundant molecule in the interstellar medium and thus has special importance. It is generally conjectured that formaldehyde and methanol are formed by the consecutive hydrogenation reactions of $\mathrm{H}$ atoms with $\mathrm{CO}$ on dust grains (Tielens \& Allamandola 1987):

$$
\mathrm{CO} \rightarrow \mathrm{HCO} \rightarrow \mathrm{H}_{2} \mathrm{CO} \rightarrow \mathrm{H}_{2} \mathrm{COH} \rightarrow \mathrm{CH}_{3} \mathrm{OH}
$$

However, no evidence for the formation of formaldehyde and methanol from the reactions of thermalized $\mathrm{H}$ atoms with $\mathrm{CO}$ at cryogenic temperatures has been obtained. Figure 3 shows the thermal desorption mass spectra for the sample of $\mathrm{CO}$ film reacting with $\mathrm{H}$ atoms at $10 \mathrm{~K}$. The peaks appearing at $110 \mathrm{~K}$ and $160 \mathrm{~K}$ in the figure just correspond to the formation of $\mathrm{H}_{2} \mathrm{CO}$ and $\mathrm{CH}_{3} \mathrm{OH}$, respectively.

It should be noted here that the yields of the products were very low (see also the latter section). This may be due to the large energy barrier for the first-step reaction $\mathrm{H}+\mathrm{CO} \rightarrow \mathrm{HCO}$.

\subsection{Reaction of $\mathbf{H}$ atoms with solid acetone, 2-propanol and ace- tonitrile}

It is worthwhile noting that the conversion of $\mathrm{H}_{2} \mathrm{CO}$ to $\mathrm{CH}_{3} \mathrm{OH}$ in the reaction $\mathrm{H}+\mathrm{CO}$ is very low despite of long-time $\mathrm{H}$-atom spray over the $\mathrm{CO}$ solid sample (yield of $\mathrm{CH}_{3} \mathrm{OH}$ is less than $20 \%$ of $\mathrm{H}_{2} \mathrm{CO}$ ). It seems likely that the reactivity 


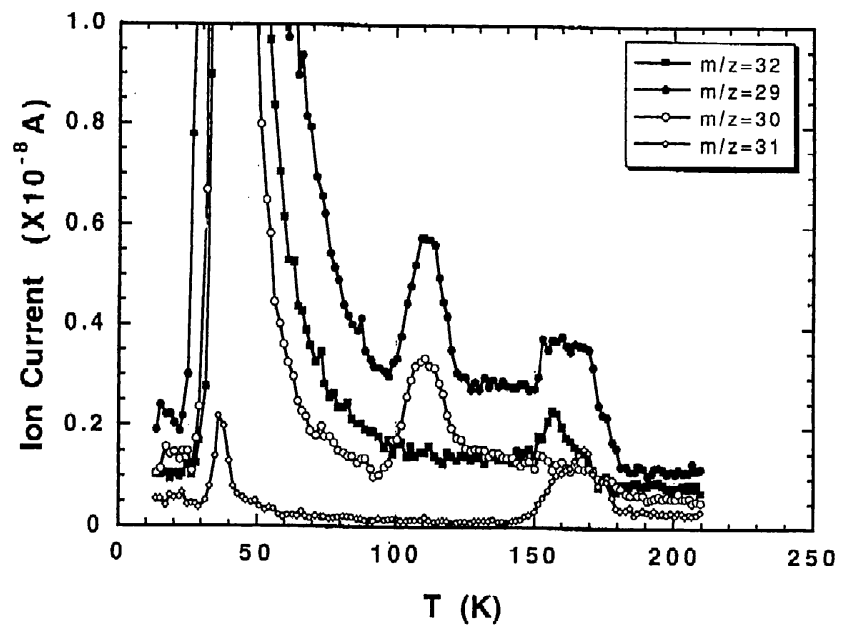

Figure 3. Temperature-programmed mass spectra of peaks with $\mathrm{m} / \mathrm{z}=29,30$, and 31 for $\mathrm{H}$-atom sprayed $\mathrm{CO}$ film at $10 \mathrm{~K}$.

of the carbonyl bond $(\mathrm{C}=\mathrm{O})$ of formaldehyde is rather low toward $\mathrm{H}$ atoms. In order to obtain more information on the reactivity of the carbonyl bond, the reaction of $\mathrm{H}$ with acetone was examined at $13 \mathrm{~K}$. The $\mathrm{H}$ atom addition reaction to the carbonyl would give 2-propanol as a reaction product. Interestingly, however, no 2-propanol was detected as a reaction product. This result is in line with the finding that the conversion of $\mathrm{H}_{2} \mathrm{CO}$ to $\mathrm{CH}_{3} \mathrm{OH}$ is low in the reaction of $\mathrm{H}$ with solid $\mathrm{CO}$. In contrast, the reactions of $\mathrm{H}$ with 2-propanol gave acetone as one of the major products. That is, the $\mathrm{H}$ atoms play not only a role in the formation of fully hydrogenated compounds through the H-atom addition reactions but also for the destruction of complex molecules formed on the dust grains through the hydrogen abstraction reactions followed by the disproportionation reactions.

\subsection{Reactions of $\mathrm{H}$ atoms with solid $\mathrm{C}_{2} \mathrm{H}_{2}$ and $\mathrm{C}_{2} \mathrm{H}_{4}$}

Cometary nuclei are the least modified solar system bodies remaining from the time of planetary formation. Their current compositions are thought to be representative of the materials from which they formed (Mumma et al. 1996). In the observations of comets Hyakutake and Hale-Bopp, it should be noted that $\mathrm{C}_{2} \mathrm{H}_{4}$ has not been detected by infrared spectroscopy despite the reasonably strong intensities of $\mathrm{C}_{2}$ hydrocarbons, $\mathrm{C}_{2} \mathrm{H}_{2}$ and $\mathrm{C}_{2} \mathrm{H}_{6}$ (Crovisier et al. 1997, Crovisier 1998; Tokunaga et al. 1996). Because $\mathrm{C}_{2} \mathrm{H}_{2}$ is known to be formed by gas-phase reactions, the $\mathrm{C}_{2} \mathrm{H}_{2}$ observed in comets is likely to have originated from the gas-phase reactions, followed by accretion on grains. In contrast, the saturated hydrocarbons cannot be formed by gas-phase reactions. The presence of $\mathrm{C}_{2} \mathrm{H}_{6}$ is consistent with production of $\mathrm{C}_{2} \mathrm{H}_{6}$ in icy grain mantles in the natal cloud, either by photolysis of $\mathrm{CH}_{4}$-rich ice (Gerakines, Schutte, \& Ehrenfreund 1996) or by hydrogen-addition reactions to $\mathrm{C}_{2} \mathrm{H}_{2}$ condensed from the gas phase. The models of gas-grain chemistry in dense interstellar clouds of Hasegawa, Herbst, \& Leung 


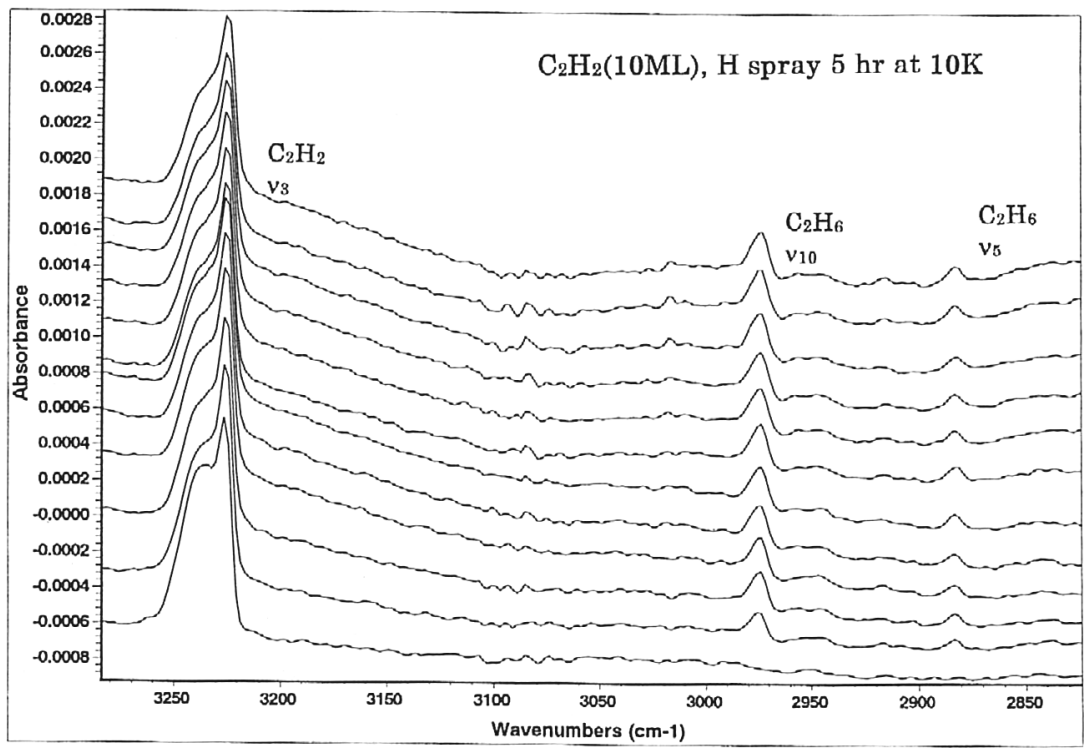

Figure 4. The change of the FT-IR spectra when the $10 \mathrm{ML}$ thick $\mathrm{C}_{2} \mathrm{H}_{2}$ film reacted with $\mathrm{H}$ atoms for 5 hours at $10 \mathrm{~K}$.

(1992) predicted that the grain surface concentrations of $\mathrm{C}_{2} \mathrm{H}_{2}$ and $\mathrm{C}_{2} \mathrm{H}_{6}$ are considerably greater than that of $\mathrm{C}_{2} \mathrm{H}_{4}$. This result is derived from the assumed larger activation energy for $\mathrm{H}+\mathrm{C}_{2} \mathrm{H}_{2}(1210 \mathrm{~K})$ than that for $\mathrm{H}+\mathrm{C}_{2} \mathrm{H}_{4}(750$ $\mathrm{K})$. Recently, Villa et al. (1998a,b) studied theoretically the tunneling reaction of $\mathrm{H}+\mathrm{C}_{2} \mathrm{H}_{4} \rightarrow \mathrm{C}_{2} \mathrm{H}_{5}$. They predicted a classical barrier height in the range 604-1360 K. Knyazev et al. (1996a,b) performed an experimental and theoretical study of the tunneling reaction $\mathrm{H}+\mathrm{C}_{2} \mathrm{H}_{2} \rightarrow \mathrm{C}_{2} \mathrm{H}_{3}$ and recommended the value of the classical barrier for the reaction to be $2014 \mathrm{~K}$.

In the present work, a systematic study of the reactions of $\mathrm{H}$ atoms with $\mathrm{C}_{2} \mathrm{H}_{2}$ and $\mathrm{C}_{2} \mathrm{H}_{4}$ has been made by means of thermal desorption and FT-IR spectroscopy in order to clarify the solid-phase chemical reactions taking place on the nuclei of comets.

Figure 4 displays the change of the FT-IR spectra when the 10 monolayer (ML) thick $\mathrm{C}_{2} \mathrm{H}_{2}$ film reacted with $\mathrm{H}$ atoms for $5 \mathrm{hrs}$ at $10 \mathrm{~K}$. The bottom spectrum is that for the $10 \mathrm{ML}$ thick $\mathrm{C}_{2} \mathrm{H}_{2}$ film before reaction. The spectra from the bottom to the top were measured with a time interval of about 25 min. Steady growth of the $\mathrm{C}_{2} \mathrm{H}_{6}$ absorption bands can be seen with the $\mathrm{H}$-atom spray time. This result may be the first in-situ observation of the synthesis of the interstellar molecule by tunneling reactions at $\sim 10 \mathrm{~K}$. In the figure, no absorption due to $\mathrm{C}_{2} \mathrm{H}_{4}$ is observed. This indicates that the reaction of $\mathrm{H}$ with $\mathrm{C}_{2} \mathrm{H}_{2}$ to form $\mathrm{C}_{2} \mathrm{H}_{3}$ is the rate-controlling process and the following reactions to form the final product $\mathrm{C}_{2} \mathrm{H}_{6}$ proceed much faster than the initial one. This finding is in accord with the observation of comets Hyakutake and Hale-Bopp, i.e., $\mathrm{C}_{2} \mathrm{H}_{2}$ and $\mathrm{C}_{2} \mathrm{H}_{6}$ but not $\mathrm{C}_{2} \mathrm{H}_{4}$ were detected in the coma of these comets.

Figure 5 displays the relationship between the yields of $\mathrm{C}_{2} \mathrm{H}_{6}$ (in \%) and the reaction temperature for reactions $\mathrm{H}+\mathrm{C}_{2} \mathrm{H}_{4}$ and $\mathrm{H}+\mathrm{C}_{2} \mathrm{H}_{2}$. It also shows 


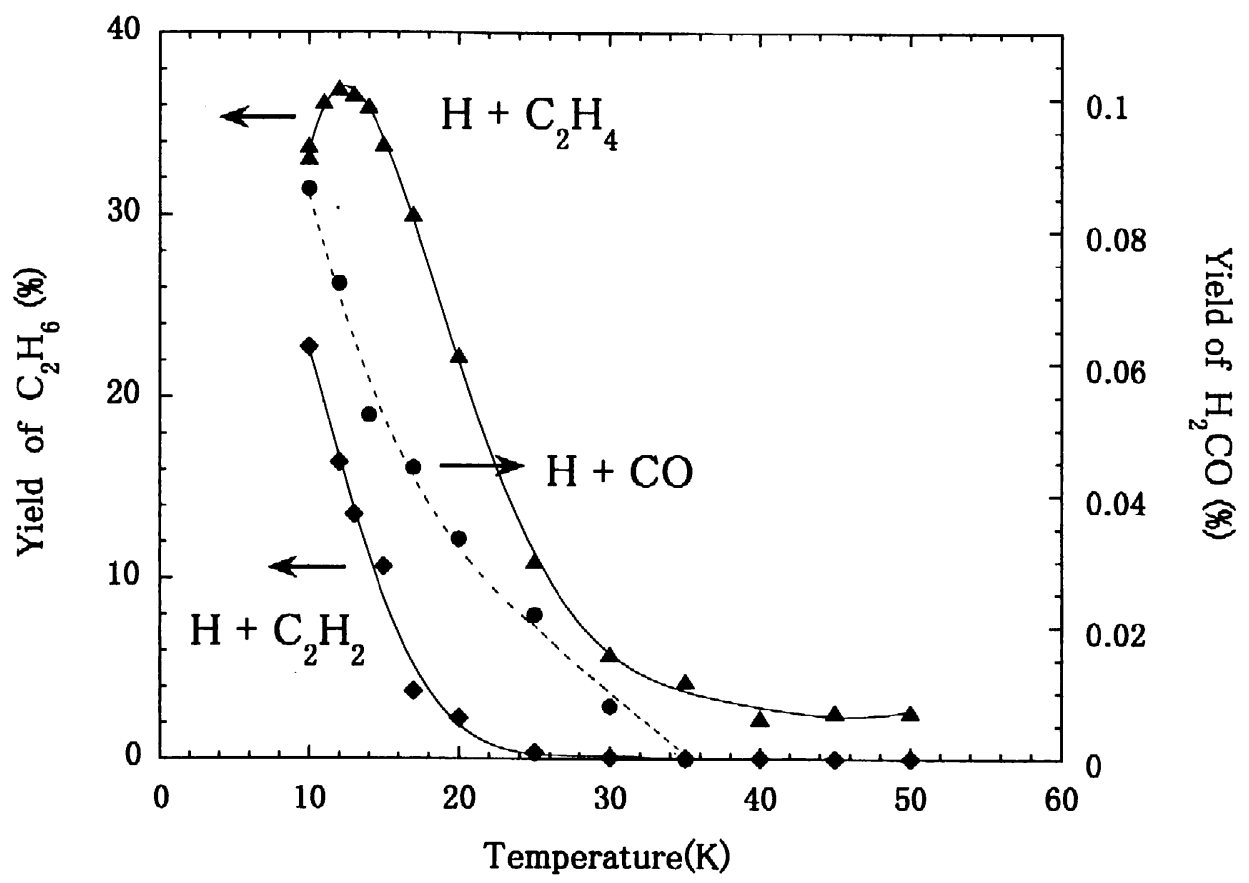

Figure 5. Temperature dependence of the yields of reaction products.

the yield of $\mathrm{H}_{2} \mathrm{CO}$ from the reaction of $\mathrm{H}+\mathrm{CO}$. For reaction $\mathrm{H}+\mathrm{C}_{2} \mathrm{H}_{4}$, the yield (\%) is temperature-independent in the range of $50-40 \mathrm{~K}$ but it shows a sharp increase below $40 \mathrm{~K}$. The yield reaches a maximum (38\%) at $12 \mathrm{~K}$ and decreases below $12 \mathrm{~K}$. For the reaction $\mathrm{H}+\mathrm{C}_{2} \mathrm{H}_{2}$, no reaction product could be detected at $50 \mathrm{~K}$. With decreasing temperature, the yield of $\mathrm{C}_{2} \mathrm{H}_{6}$ shows a steep increase below $25 \mathrm{~K}$. The unprecedented negative temperature dependence of the product yields in Figure 5 is very unique because the rate constants of the chemical reactions generally have a positive temperature dependence. This finding may be explicable qualitatively in that the sticking probability of $\mathrm{H}$ atoms to the solid sample surface increases at lower temperature.

The yield of $\mathrm{H}_{2} \mathrm{CO}$ also shows a steep negative temperature dependence in Figure 5. It should be noted that the yield of $\mathrm{H}_{2} \mathrm{CO}$ at $10 \mathrm{~K}(0.09 \%)$ is much lower than that of $\mathrm{C}_{2} \mathrm{H}_{6}$ from the reactions $\mathrm{H}+\mathrm{C}_{2} \mathrm{H}_{2}$ and $\mathrm{H}+\mathrm{C}_{2} \mathrm{H}_{4}$. It is evident that the $\mathrm{H}$ atom is much less reactive toward $\mathrm{CO}$ than $\mathrm{C}_{2}$ unsaturated hydrocarbons.

The reactivities of $\mathrm{H}$ with molecules with $\pi$ bonds can be predicted by quantum chemistry. Since the $\mathrm{H}$ atom is a radical, it must interact with both $\pi$ and $\pi^{*}$ orbitals of the unsaturated reactant in the first-step addition reaction. The interaction becomes more favorable for the reactant which has a smaller energy gap between the $\pi$ and $\pi^{*}$ orbitals. The order of $\pi-\pi^{*}$ energy gaps is $\mathrm{C}_{2} \mathrm{H}_{4}<$ carbonyl $(\mathrm{C}=\mathrm{O})<\mathrm{C}_{2} \mathrm{H}_{2}<$ cyano-bond(-CN). This explains the trend observed in the present experiments. 
The negative temperature dependence of the $\mathrm{H}$-atom sticking probability to the solid surface would result in a steep increase of the $\mathrm{H}$-atom concentration on the grain surface at lower temperature which leads to the efficient chemical evolution on the dust grains via tunneling reactions. The fact that the grain temperature is kept at about $10 \mathrm{~K}$ is very important for the chemical evolution in dark clouds. In interstellar clouds where the temperature is higher than 10 $\mathrm{K}$, the chemical evolution via $\mathrm{H}$-atom tunneling reactions would be much slower due to the short residence time of the $\mathrm{H}$ atoms on the dust grains.

\section{References}

Brown, P.D. \& Charnley, S.B. 1990, MNRAS, 244, 432

Crovisier, J. 1998, Faraday Discuss., 109, 437

Crovisier, J., Leech, K., Bockelée-Morvan, D., Brooke, T.Y., Hanner, M.S., Altieri, B., Keller, H.U. \& Lellouch, E. 1997, Science, 275, 1904

d'Hendecourt, L.B., Allamandola, L.J., \& Greenberg, J.M. 1985, A\&A, 152, 130 Gerakines, P.A., Schutte, W.A., \& Ehrenfreund, P. 1996, A\&A, 312, 289

Greenberg, J.M. \& Li, A. 1996, A\&A, 309, 258

Greenberg, J.M. \& Pirronello, V. 1989, Chemistry in Space, NATO ASI Series, Kluwer Academic, Dordrecht

Hasegawa, T.I., Herbst, E., \& Leung, C.M. 1992, ApJ, 82, 167

Hiraoka, K., Ohashi, N., Kihara, Y., Yamamoto, K., Sato, T., \& Yamashita, A. 1994, Chem. Phys. Lett., 229, 408

Hiraoka, K., Miyagoshi, T., Takayama, T., Yamamoto, T., \& Kihara, Y. 1998a, ApJ, 498, 710

Hiraoka, K., Yamamoto, K., Kihara, Y., Takayama, T., \& Sato, T. 1999, ApJ, 514,524

Hiraoka, K., Yamashita, A., Miyagoshi, T., Ohashi, N., Kihara, Y., \& Yamamoto, K. 1998b, ApJ, 508, 423

Hiraoka, K., Yamashita, A., Yachi, Y., et al. 1995, ApJ, 443, 363

Hong, S.S. \& Greenberg, J.M. 1980, A\&A, 309, 194

Ikezoe, Y., Matsuoka, S., Takebe, M., \& Viggiano, A. 1987, Gas Phase Ion-

Molecule Reaction Rate Constants Through 1986, Maruzen, Tokyo

Kaiser, R.I., Eich, G., Gabrysch, A., \& Roessler, K. 1997, ApJ, 484, 487 1999, A\&A, 346, 340.

Kaiser, R.I. \& Roessler, K. 1997, ApJ, 475, 144 1998, ApJ, 503, 959

Knyazev, V.D., Bencsura, A., Stoliarov, S.I., \& Slagle I.R. 1996b, J. Phys. Chem., 100, 11346

Knyazev, V.D. \& Slagle, I.R. 1996a, J. Phys. Chem., 100, 16899

Mumma, M.J., DiSanti, M.A., Russo, N.D., Fomenkova, M., Magee-Sauer, K., Kaminski, C.D., \& Xie, D.X. 1996, Science, 272, 1310

Pirronello, V., Biham, O., Liu, C., Shen, L., \& Vidali G. 1997b, ApJ, 483, L131

Pirronello, V., Liu, C., Shen, L., \& Vidali, G. 1997a, ApJ, 475, L69

Pirronello, V., Liu, C., Roser, J.E., \& Vidali, G. 1999, A\&A, 344, 681

Tielens, A.G.G.M. \& Allamandola, L.J. 1987, Interstellar Processes, eds. D.J.

Hollenbach \& H.A. Thronson (Reidel: Dordrecht)

Tielens, A.G.G.M. \& Hagen, 1982, A\&A, 114, 245

Tokunaga, T., Brooke, T.Y., Weaver, H.A., Crovisier, J., \& Bockelée-Morvan, D. 1996, IAU Circ., 6378 
Villa, J., Corchado, J.C., Gonzalez-Lafont, A., Lluck, J.M., \& Truhlar, D.J. 1998a, J. Am. Chem. Soc., 120, 12141

Villa, J., Gonzalez-Lafont, A., Lluck, J.M., \& Truhlar, D.J. 1998b, J. Am. Chem. Soc., 120,5559

Williams, D.A. \& Millar, T.J. 1993, Dust and Chemistry in Astronomy, IOP, Bristol

\section{Discussion}

H. J. Fraser: (1) Can you comment on the thickness of your molecular ice layer on the silicon substrate? Is it thick enough to render the silicate substrate inert? If not, how similar to the silicate core in dust sand is your wafer? (2) Secondly what effect does the silicon heating rate have on the production of $\mathrm{HCOOH}$ and $\mathrm{CH}_{3} \mathrm{OH}$ ? What is the heating rate in the desorption experiment?

K. Hiraoka: (1) S, N, and O atoms were not deposited on the ice film but were co-deposited with $\mathrm{CO}, \mathrm{N}_{2}$, and $\mathrm{N}_{2} \mathrm{O}$ molecules, respectively, on the silicon substrate. The procedure of the $\mathrm{H}$ atom spray over the $10 \mathrm{ML}$ thick film was repeated 100 times in order to accumulate the products. Since the reactions take place mainly near the surface of the film, the reactions may not be affected by the surface conditions of the substrate. (2) The programming rate of the silicon substrate was $0.8 \mathrm{~K} \mathrm{~min}^{-1}$. This is the maximum value for our apparatus due to the limited capacity of the heater used. A smaller heating rate would reduce the resolution of the thermal desorption spectra.

D. A. Williams: In the hydrogenation of $\mathrm{C}, \mathrm{N}$, and $\mathrm{O}$ is there any evidence of desorption of the saturated products in the absence of temperature programmed desorption?

$K$. Hiraoka: If the $\mathrm{H}$ atom and some radical recombine with each other, a considerable amount of heat must be involved and local heating may occur. However, no sign of any desorption of the products was detected by the quadrupole mass spectrometer. If one assumes that the quadrupole mass spectrometer is sensitive enough to detect the desorbed products, this result may mean that the relaxation of the heat involved is rapid enough to cool down the hot products. The molecular matrices seem to act as an excellent heat bath. This may not be the case for the rare gas matrices.

$W$. A. Schutte: In your original paper on the hydrogenation of $\mathrm{CO}$ you proposed that the low yield of $\mathrm{H}_{2} \mathrm{CO}$ and $\mathrm{CH}_{3} \mathrm{OH}$ could be due to the hydrogen abstraction reaction $\mathrm{HCO}+\mathrm{H} \rightarrow \mathrm{CO}+\mathrm{H}_{2}$. In this case you would expect a high yield of $\mathrm{HCO}$ relative to $\mathrm{H}_{2} \mathrm{CO}$ and $\mathrm{CH}_{3} \mathrm{OH}$ in your experiments. Do in-situ infrared measurements indicate a high production of $\mathrm{HCO}$ from surface hydrogenation of $\mathrm{CO}$ ?

K. Hiraoka: No $\mathrm{HCO}$ absorption was observed in the reaction of $\mathrm{H}$ with solid $\mathrm{CO}$ at $10 \mathrm{~K}$. This suggests that the rate-controlling step for the formation of $\mathrm{HCHO}$ from the reaction of $\mathrm{H}+\mathrm{CO}$ is not $\mathrm{H}+\mathrm{HCO} \rightarrow \mathrm{H}_{2}+\mathrm{CO}$ but $\mathrm{H}+\mathrm{CO}$ $\rightarrow \mathrm{HCO}$. In fact, we found that the $\mathrm{H}$ atoms have a long diffusion length in the CO matrix. 